\title{
Identification of Distillation Process Dynamics Comparing Process Knowledge and Black Box Based Approaches
}

\author{
Rasmussen, Knud H; Nielsen, C. S.; Jørgensen, Sten Bay
}

Published in:

American Control Conference

Publication date:

1990

Document Version

Publisher's PDF, also known as Version of record

Link back to DTU Orbit

Citation (APA):

Rasmussen, K. H., Nielsen, C. S., \& Jørgensen, S. B. (1990). Identification of Distillation Process Dynamics Comparing Process Knowledge and Black Box Based Approaches. In American Control Conference (pp. 31163121). IEEE.

\section{General rights}

Copyright and moral rights for the publications made accessible in the public portal are retained by the authors and/or other copyright owners and it is a condition of accessing publications that users recognise and abide by the legal requirements associated with these rights.

- Users may download and print one copy of any publication from the public portal for the purpose of private study or research.

- You may not further distribute the material or use it for any profit-making activity or commercial gain

- You may freely distribute the URL identifying the publication in the public portal 


\title{
TP1 2:30
}

\section{Identification of Distillation Process Dynamics Comparing Process Knowledge and Black Box Based Approaches.}

\author{
K.H. Rasmussen C.S. Nielsen S. Bay Jørgensen \\ The Proceas Deaign, Dynamics, and Control Group \\ Institut for Kemiteknil, Technical University of Denmark, DK-2800 Lyngby, Denmark
}

\begin{abstract}
A distillation plant equipped with a heat pump separates a mixture of isopropanol and methanol. The mixture contains some water as impurity. The model development aims at dual composition control design, where top and bottom compositions should follow the setpoints, and disturbances should be rejected. Disturbances may oecur in feed flow rate and feed composition.

Identification is performed using multivariable linear discrete time model structure development tools: a process knowledge based and a black box approach. In the process knowledge based approach, the model structure is developed from qualitative process knowledge which presently may require modification to guarantee identifiability. The black box approach is based on pseudocanonical MFD model representation, where the model structure is determined by a set of structure indices. The identifications are performed on experimental data obtained in closed loop operation of the distillation plant.

In the present work, the two approaches are compared in terms of how well the model fits, and predicts the data, the conditioning of the model parameter estimation, and convenience of nsage.
\end{abstract}

\section{Introduction}

Process identification is a disciplin for development of process model structures and estimation of parameter values. The process models may be applied for control design for processes with complex dynamic behaviour. Such processes may require multivariable control design in order to minimise loop interactions and to reduce the effects of complex dynamics. In the present paper, the main focus is therefore on development of multivariable process models.

Model development for process control can be considered as a three stage procedure:

1. selection of measurements and actuators

2. determination of model structure

3. determination of model parameters and model validation

The first stage is usually performed using qualitative knowledge about static and dynamic process measurement and actuator sensitivities. This first stage is considered completed in the present study where data are used which have been collected during closed loop identification experiments on a nearly binary distillation column with an indirect heat pump. The main subject of the present paper is on the two latter stages. The model structure selection is performed using two different approaches. One approach is a black box approach [4] using a pseudocanonical model structure, where the structure indices are evaluated based upon the conditioning of the parameter estimation and the ability to fit and predict data. In the second approach [8], the model structure is formulated and based upon qualitative process knowledge, similar to that used in stage one above. The model complexity is selected based upon the ability to predict and fit data. The purpose of the present paper is to investigate and elucidate the relative merits of the two approaches on a binary distillation plant with an indirect heat pump.

First the two model structure selectioning approaches are presented in section 2 . Then the process plant and experimental conditions are given in section 3 . The results are presented in section 4 . Then in section 5 , the relative merits of the two approaches are discussed.

\section{Model Structures}

This section contains a brief introduction to the blackbox and the process knowledge based methods used for obtainaining a model structure for identification. The models obtained are linear discrete time MIMO models.

\subsection{Black Box Method}

The parametrisation used for identification is the pseudocanonical observable (p.c.o) parametrisation. 
This parametrisation is throughly discussed by Gevers and Wertz [4].

\subsubsection{The Parametrisation}

The p.c.o model structure is uniqly determined by a set of structure indices: $n_{1}, n_{2} \ldots n_{p}$ where $p$ is the number of outputs. The sum of the $p$ structure indices is the order of the system: $n=\sum_{i=1}^{p} n_{i}$. Given the structure indices, a state space model is easily obtained:

$$
\begin{aligned}
\mathbf{x}(t+1) & =\mathbf{A} * \mathbf{x}(t)+\mathbf{B} * \mathbf{u}(t) \\
\mathbf{y}(t) & =\mathbf{C} * \mathbf{x}(t)
\end{aligned}
$$

where $\mathbf{A}$ contains block matrices.

$$
\mathbf{A}=\left(\begin{array}{ccc}
\mathbf{A}_{11} & \cdots & \mathbf{A}_{1 p} \\
\vdots & & \vdots \\
\mathbf{A}_{p 1} & \cdots & \mathbf{A}_{p p}
\end{array}\right)(n \times n)
$$

where the blocks are:

$$
\begin{aligned}
& \mathbf{A}_{i i}=\left(\begin{array}{ccc}
0 & & \\
\vdots & I_{n_{i} 1} & \\
0 & & \\
a_{i i 1} & \cdots & a_{i i n_{i}}
\end{array}\right) \quad\left(n_{i} \times n_{i}\right) \\
& \mathbf{A}_{i j}=\left(\begin{array}{ccc} 
& \\
& 0 & \\
a_{i j 1} & \cdots & a_{i j n_{j}}
\end{array}\right) \quad\left(n_{i} \times n_{j}\right)
\end{aligned}
$$

$\mathbf{B}$ is a fully parametrisised $p \times m$ matrix where $m$ is the number of inputs. $C$ is a $p \times n$ matrix containing zeros, except one element in each row. In the $j$ 'th row, element

$$
q_{j}=1+\sum_{i=1}^{j 1} n_{i}
$$

is equal to one. It is easy to see that the p.c.o parametrisation contains $n \times(p+m)$ parameters, this is a little more than a canonical parametrisation which contains up to $n \times(p+m)$ parameters, but the p.c.o parametrisation is still identifiable. Given a MIMO linear system of order $n$, then it can be transformed into different p.c.o parametrisations (i.e. parametrisations with a different set of structure indices), the p.c.o parametrisations are overlapping. This is not the case with canonical parametrisations, where only a MIMO linear system can be transformed into one canonical parametrisation determined by the observability indices of the system.

\subsubsection{Structure Estimation}

Using p.c.o parametrisations for identification, only the $p$ structure indices are needed to define the model structure. Since a system can be represented in different p.c.o parametrisations, it is not critical that the structure indices are chosen correctly. Van Overbeek and Ljung [12] suggested that identification started using one parametrisation, and then the parametrisation may be changed on-line if the parameter estimation became ill conditioned. Perez-Côrrea and Kershenbaum [11] used p.c.o state space models in a multivariable selftuning controller, and pointed out that the selection of the structure indices was not critical. Numerous groups have worked with the problem of determining a set of structure indices which will give a good or the best model, Gevers and Wertz [4]. A method which is often used selects the structure indices such that the parameter estimation becomes well conditioned $[2,5,13]$.

\section{1.s Input Output Models}

For parameter estimation it is often convenient to use input output models. The p.c.o state space model is easily transformed to a matrix fraction description (MFD):

$$
\mathbf{P}(\mathbf{q}) * \mathbf{y}(\mathbf{t})=\mathbf{Q}(\mathbf{q}) \bullet \mathbf{u}(\mathbf{t})
$$

where $q$ is forward shift operator $P(q)$ and $Q(q)$ are polynominal matrices:

$$
\begin{aligned}
& \mathbf{P}(q)=\left(\begin{array}{ccc}
P_{11}(q) & \cdots & P_{1 p}(q) \\
\vdots & & \vdots \\
P_{p 1}(q) & \cdots & P_{p p}(q)
\end{array}\right)(p \times p) \\
& \mathbf{Q}(q)=\left(\begin{array}{ccc}
Q_{11}(q) & \cdots & Q_{1 m}(q) \\
\vdots & & \vdots \\
Q_{p 1}(q) & \cdots & Q_{p m}(q)
\end{array}\right)(p \times m) \\
& P_{i i}=q^{n_{i}}-a_{i i n_{i}} q^{n_{i 1}}-\cdots-a_{i i 2} q-a_{i i 1} \\
& P_{i j}=-a_{i j n_{j}} q^{n_{j} 1}-\cdots-a_{i j 2} q-a_{i j 1} \\
& Q_{i j}=b_{i j r_{i}} q^{r i 1}+\cdots+b_{i j 2} q+b_{i j 1} \\
& r_{i}=\max \left(r, n_{i}\right) \quad r=\max \left(n_{j}\right)-1
\end{aligned}
$$

From this it is seen that if $\max \left(n_{j}-n_{k}\right) \geq 2$ then the number of parameters in the p.c.o MFD is greater than $n \times(p+m)$. This means that all the parameters in the MFD are not independent. Corrêa and Glover [3] show that if the outputs are permuted such that the structure indices are arranged in decreasing order $n_{1} \geq n_{2} \geq \ldots \geq n_{p}$ then if the parameters are estimated for each output, starting with the output with the highest structure index, the redundant parameters can be expressed in terms of the parameters estimated on the lines above. If the constraints on the parameters are not taken into account, the MFD will not be proper. The p.c.o MFD's are not in general monic, only p.c.o MFD's where the structure indices are equal $\left(n_{1}=n_{2}=\cdots=n_{p}\right)$ are monic. For monic p.c.o. models the present output can be expressed in terms of previous in- and outputs:

$$
\mathbf{y}(t+1)=\mathbf{A}\left(q^{1}\right) * \mathbf{y}(t)+\mathbf{B}\left(q^{1}\right) * u(t)
$$




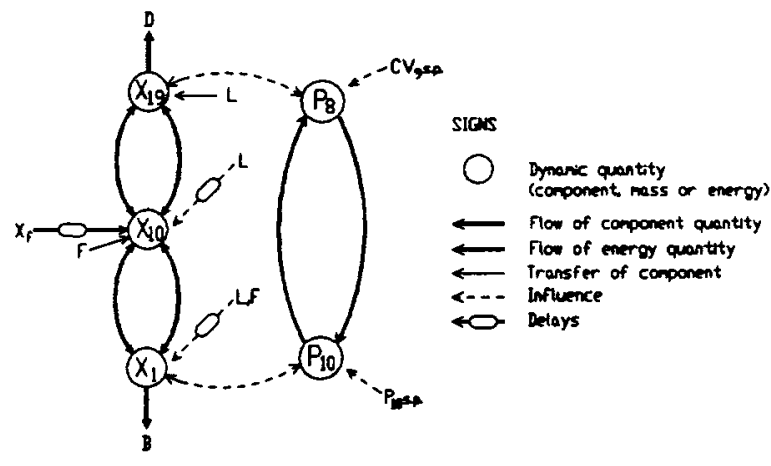

Figure 1: Dyquid for a distillation column

2.2 Physical Knowledge Based Method The model structures obtained from physical knowledge are obtained from qualitative models. The method used here is the DYQUID method $[7,8]$.

\subsubsection{The DYQUID Method}

The DYQUID (DYnamic QUantity Interaction Diagram) method uses a priori process knowledge such as coupling between states, interactions, and flows to construct a diagram that visualizes the interaction between the relevant quantities as well as the dynamics in the process. Important dynamic quantities such as component mass and energy, quantity influence and transfers, and the variables which influence the rate of flows/transfers are included in this type of diagram.

\subsubsection{DYQUID for a Distillation Column} A schematic DYQUID of the binary separation in the column and heat pump is shown in figure 1 , Nielsen et al. [10].

\subsubsection{Input Output Models}

A discrete time multivariable input-output model structure is developed proceeding through the following sequence: First a continous time state space model structure is derived from the DYQUID. Therefrom a discrete time model structure is obtained. Subsequentially, the model structure is transformed to an input-output form including only the measured outputs. Thus a discrete time ARX model structure has been obtained. The ARX model obtained from the DYQUID is monic. The structure of monic ARX models is not yet understood [4], therefore some parameters might be redundant. For on-line estimation of the unknown model parameters a first order moving average term is often included for each output to yield an ARMAX model. In this work, all estimation is off-line thus only the ARX structure is applied. The ARX model structure is used as a vehicle for a search procedure for a perhaps improved model structure. A tool for an improved model structure is to investigate if some parameters are strongly correlated or uncertain.

\subsection{Model Validation}

The following four types of measure are used for model validation in this work:

- model misfit to data used for estimation.

- model misfit to data not used for estimation.

- condition of parameter estimation.

- multivariable frequency domain properties.

The first two measures are based upon the model misfit for the i'th output $y_{i}(t)$ :

$$
R_{i}=\frac{\operatorname{var}\left(e_{i}(t)\right)}{\operatorname{var}\left(y_{i}(t)\right)} \text { where } e_{i}(t)=y_{i}(t)-\hat{y}_{i}(t)
$$

where $\hat{y}_{i}$ is the one step prediction of output $i$. If $R_{i}$ is close to sero, the model fits the data well. The third measure is applied to indicate if a too high model order is being approached. A too high model order would make the parameter estimation ill-conditioned and render the model less able to predict future dynamics (measure two). Using a LS procedure the condition number of the parameter covariance matrix is a measure of the conditioning of the parameter estimation:

$$
K_{i}=\frac{\sigma_{\max , i}}{\sigma_{\min , i}}
$$

where $\sigma_{m a x, i}$ and $\sigma_{\min , i}$ are the largest and smallest singular value of the parameter covariance matrix. If the purpose of the model is that it should be used for multivariable control, we must demand that the model describes the multivariable structure of the plant and the gain directionality. A tool for investigating this is multivariable frequency domain anat ysis, Andersen et al. [1].

\section{Experiment}

\subsection{Experimental Setup}

The study is carried out on a full scale distillation setup at Institut for Kemiteknik. Figure 2 shows a simplified process diagram. The plant consists of three major parts: the column, a heat pump section, and a tank park. Details are given by Hallager et al. [6]. Separation of methanol and isopropanol with some water impurity is carried out in the 19 tray column which is $10.5 \mathrm{~m}$ high and has an internal diameter of $0.47 \mathrm{~m}$. Trays are numbered from bottom to top starting with no. 1 . Feed is pumped from the tank park as two feedstreams F1 and F2 which are mixed before the feed heater and enters the column at tray no. 10. Piping and feed heat exchanger between the 


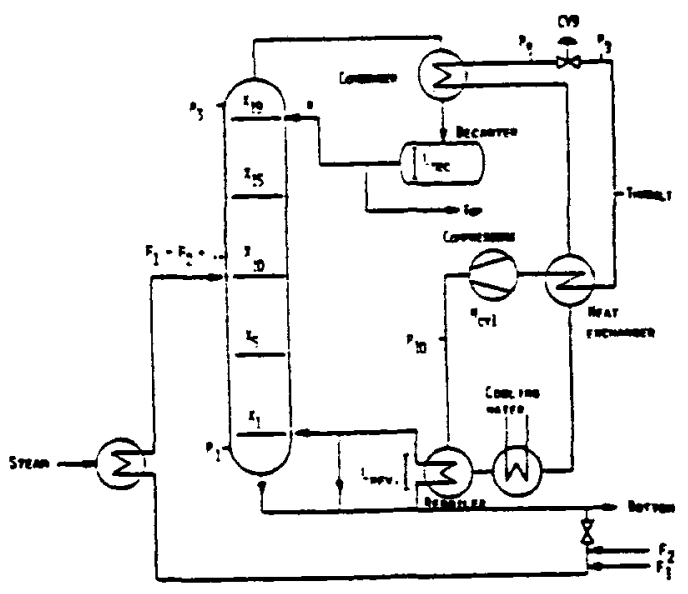

Figure 2: Process diagram

$\begin{array}{llrl}\text { Namber of cylinders } & \text { Ncyl } & 10 & \\ \text { High pressure in heat pump } & \text { P10 } & 11.0 & \text { bar } \\ \text { Low pressure } & \text { P8 } & 4.0 & \text { bar } \\ \text { Pressure drop P8-P9 } & \Delta P & 1.0 & \mathrm{bar} \\ \text { Reflux rate } & \mathrm{R} & 15.0 & \mathrm{l} / \mathrm{min} \\ \text { Feedflow } & \mathrm{F} & 4.5 & \mathrm{l} / \mathrm{min} \\ \text { Feed concentration } & \mathrm{X}_{f} & 0.5 & \end{array}$

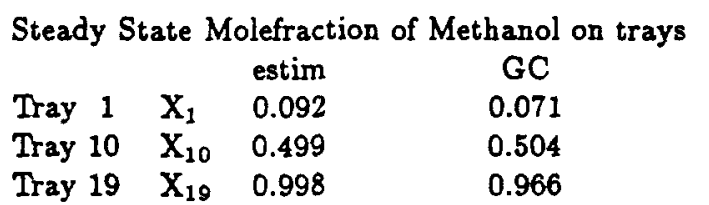

Table 1: Experimental operation point

mixing point and column entrance contain approx. 54 1 of liquid. Some mixing will occur in the end sections of the heater, these volumes are around $8 \mathrm{l}$ each. The condensed vapor is pumped to the accumulator from which the reflux and top products are taken. At the bottom the column is coupled to a thermosiphon reboiler producing the vapor flow. Bottom product is also withdrawn here. Distillation products are led to the tank park.

The indirect heat pump recirculates the energy from vapor condensation in the condenser to heating in the reboiler. Essentially, Freon R-114 is evaporated in the condenser, compressed to about 10 bar and condensed in the reboiler. For this purpose two 8-cylinders piston flow compressors are employed. The freon loop is closed.

Data used in this study are collected around the operation point given in table 1 . Concentrations are molefraction methanol estimated, based on temperatures measured using PT-100 sensors and column pressure at the tray in question assuming an ideal binary mixture. In addition, samples are analysed off-line on a HP-gaschromatograph. We refer to Nielsen et al. [9] for further details.

\subsection{Location of Measurements}

Selection of no. and location of measurements is essential. The measurements must be representative for the important dynamic quantities. They should provide early detection of the disturbances and equally important they should be sensitive to variations in the important quantities. P8 (low pressure) in the heat pump section is chosen to represent the energy quantities around the condenser on the freon side. The transport mechanisms in the column are convection and dispersion. Convective transport may be described using three measurement points. Therefore at least three measurements are necessary for the column. Methanol concentrations on tray 1, 10, and 19 are chosen. The top and bottom concentration measurements are selected for control purposes. It is advantageous to have a measurement for the product purities which are to be controlled. The feed-tray (tray no. 10) is selected as a third location because the disturbance enters the column here. Also this concentration measurement is very sensitive to variations in the mass of a component in the column because the profile is rather steep in the middle of the column. Additional concentration measurements, if necessary should be located in the center of each section of the column, where the distance between the working line and the equilibrium curve is large yielding maxima in sensitivity.

\subsection{Perturbation Experiment}

A perturbation experiment was carried out around the operation point shown in table 1. P10 (high pressure in the heat pump), $\Delta P$ (presure difference between $\mathrm{P} 8$ and $\mathrm{P9}$ ), $\mathrm{R}$ (reflux rate), $\mathrm{Xf}$ (mole fraction of methanol in feed) and $F$ (feed flow rate) are all controlled by SISO loops. The manipulated variables in this experiment are the setpoints to the five SISO loops. The measured variables are P8 (low preassure in heat pump), $\mathrm{X}_{1}$ (mole fraction of methanol in bottom), X10 (mole fraction of methanol at the feed tray), and X19 (mole fraction of methanol at the top tray). The variables are sampled every $2 \mathrm{~min}$.

To maintain the concentration profile in the column, it was necessary to perform the perturbation experiment in closed loop. The controller is a multivariable high order constant gain controller. To the output from the controller is added a PRBS (pseudo random binear sequence), such that the process inputs are made from the control signals given by the controller with a PRBS in addition. This is done in order to make the closed loop data informative for identification.

\section{Results}

Parameter estimation was performed on $2 / 3$ of the 290 elements in the data vector. The rest were used for validation. The results using the two methods are 


\begin{tabular}{rrrrr} 
n & Indices & $R_{3}$ & $K_{3}$ & $R_{3}$ \\
\hline 4 & $(1,1,1,1)$ & 0.157 & 67 & 0.173 \\
5 & $(1,1,2,1)$ & 0.024 & 154 & 0.038 \\
6 & $(2,1,2,1)$ & 0.021 & 183 & 0.037 \\
7 & $(2,1,2,2)$ & 0.021 & 219 & 0.038 \\
8 & $(2,2,2,2)$ & 0.021 & 402 & 0.041
\end{tabular}

Table 2: Validation measures for output $3\left(X_{10}\right)$ with different model orders, n model order, $R_{3}$ residual on data used for estimation, $K_{3}$ Condition number of parameter covariance matrix, $B_{\zeta}$ residual on data not used for identification.

$$
\begin{aligned}
\mathbf{P}_{0} & =\left(\begin{array}{rrrrr}
-0.082 & -0.039 & -0.001 & -0.008 \\
-0.057 & -0.472 & 0.541 & -0.063 \\
-0.054 & 0.024 & 0.045 & -0.104 \\
0.068 & 0.088 & -0.167 & -0.618
\end{array}\right) \\
\mathbf{Q}_{1} & =\left(\begin{array}{rrrrr}
0.003 & 0.034 & 0.033 & 0.484 & 0.319 \\
0 & 0 & 0 & 0 & 0 \\
0.027 & 0.065 & 0.148 & -0.167 & 0.127 \\
0 & 0 & 0 & 0 & 0
\end{array}\right) \\
\mathbf{Q}_{0} & =\left(\begin{array}{rrrrr}
-0.015 & -0.021 & -0.020 & 0.426 & 0.005 \\
0.001 & 0.076 & -0.036 & 0.005 & -0.025 \\
0.027 & 0.056 & 0.299 & -0.352 & 0.054 \\
0.012 & -0.020 & 0.085 & 0.025 & 0.108
\end{array}\right)
\end{aligned}
$$

discussed below. The outputs are $y=\left(P_{\mathrm{e}}, X_{1}, X_{10}, X_{19}\right)^{\text {TIt }}$ is seen that the MFD contains $6 \times(4+5)=54$ and the inputs are $u=\left(X_{F}, F, R, P_{10}, \Delta P\right)^{T}$. A change in feed concentration will occur at the feed tray about 5 samples delayed, due to the piping between the mixing point and the feed point. Therefore, this information is incorporated by letting $X_{F}$ in the input vector be 5 samples delayed.

\subsection{P.C.O Model}

Parameter estimation and validation were performed on all possible p.c.o models from order 4 to 8 . In table 2 , the validation measures for outpat $3\left(X_{10}\right)$ are shown for different p.c.o. models of different order. It is seen that $R_{3}$ is decreasing with increasing model order, and the condition number $K_{3}$ is growing because more parameters are used for identification with increasing model order. More interesting is the fact that $\boldsymbol{R}_{3}$ first is decreasing and then is increasing for $n>6$, probably because the extra parameters included for $n>6$ are not used to describe important process dynamics but noise. Inspecting similar results for other outputs and other p.c.o. models it was found that selecting a the model is not an easy task as one might conclude from table 2. By inspecting residuals and condition number for each output, a 6 order p.c.o model with structure indices $(2,1,2,1)$ was selected. It was found in this case by comparing all p.c.o models of order 6 that looking for a well conditioned parametrisation for parameter estimation does not guarantee that the model describes the data well. The estimated MFD model is:

$$
\begin{array}{r}
\mathbf{P}_{2} * \mathbf{y}(t+2)+\mathbf{P}_{1} * \mathbf{y}(t+1)+\mathbf{P}_{0} * \mathbf{y}(t) \\
=\mathbf{Q}_{1} * \mathbf{u}(t+1)+\mathbf{Q}_{0} * u(t)
\end{array}
$$

where

$$
\begin{gathered}
\mathbf{P}_{2}=\left(\begin{array}{llll}
1 & 0 & 0 & 0 \\
0 & 0 & 0 & 0 \\
0 & 0 & 1 & 0 \\
0 & 0 & 0 & 0
\end{array}\right) \\
\mathbf{P}_{1}=\left(\begin{array}{rrrr}
-0.193 & 0 & 0.089 & 0 \\
-0.041 & 1 & -0.990 & 0 \\
-0.143 & 0 & -0.875 & 0 \\
0.038 & 0 & -0.423 & 1
\end{array}\right)
\end{gathered}
$$

parameters, and that the MFD is not monic (i.e. $\mathbf{P}_{\mathbf{2}}$ is not a nnit matrix).

\subsection{DYQUID Model}

The discrete time linear ARX model constructed from the DYQUID was used for parameter estimation. The estimated ARX model is:

$$
\begin{aligned}
\mathbf{y}(t) & =\mathbf{A}_{1} * \mathbf{y}(t-1)+\mathbf{A}_{2} * \mathbf{y}(t-2) \\
& +\mathbf{B}_{1} * \mathbf{u}(t-1)+\mathbf{B}_{2} * \mathbf{u}(t-2)
\end{aligned}
$$

where

$$
\begin{aligned}
\mathbf{A}_{1} & =\left(\begin{array}{rrrrr}
0.252 & & 0 & 0 & -0.161 \\
0.182 & 0.316 & 0.436 & 0 \\
0.134 & -0.090 & 0.932 & -0.058 \\
0 & -0.189 & 0.507 & 0.622
\end{array}\right) \\
\mathbf{A}_{2} & =\left(\begin{array}{rrrrr}
0 & 0 & 0 & 0.117 \\
0 & 0 & 0 & 0.145 \\
0.059 & 0 & 0 & 0.128 \\
0.082 & 0.182 & 0 & 0
\end{array}\right) \\
\mathbf{B}_{1} & =\left(\begin{array}{rrrrr}
0 & 0 & 0 & 0.475 & 0.326 \\
0 & 0.123 & 0.062 & -0.122 & 0.124 \\
0.027 & 0.058 & 0.118 & -0.162 & 0.128 \\
0 & 0 & 0.098 & -0.022 & 0.157
\end{array}\right) \\
\mathbf{B}_{2}= & \left.\begin{array}{rrrrr}
0 \\
0 & 0 & 0 & 0.412 & 0 \\
0 & 0.126 & 0.340 & -0.401 & 0 \\
0 & 0.071 & 0.336 & -0.352 & 0.064 \\
0 & 0 & 0.209 & -0.247 & 0
\end{array}\right)
\end{aligned}
$$

The ARX model contains 42 estimated parameters. It is seen that the ARX model can be viewed as an 8 order p.c.o. model with structure indices $(2,2,2,2)$, where some parameters are fixed to sero.

\subsection{Comparing the two Methods}

As seen in table 3 the residuals are nearly equal for the outputs, but the condition number is in general larger for the p.c.o model. This is due to the fact that the p.c.o model contains slightly more parameters. The p.c.o MFD's are not monic as the ARX model obtained from the DYQUID method. This means that 


\begin{tabular}{r|rrr|rrr} 
& \multicolumn{3}{|c|}{ P.C.O. } & \multicolumn{3}{c}{ DYQUID } \\
$\#$ & $R$ & $K$ & $R$ & $R$ & $K$ & $R$ \\
\hline 1 & 0.099 & 183 & 0.147 & 0.101 & 20 & 0.141 \\
2 & 0.078 & 142 & 0.086 & 0.092 & 66 & 0.135 \\
3 & 0.021 & 183 & 0.037 & 0.021 & 158 & 0.043 \\
4 & 0.060 & 142 & 0.184 & 0.055 & 99 & 0.191
\end{tabular}

Table 3: Validation results: \# output number, $R$ residual on data used for estimation, $K$ Condition number of parameter covariance matrix, $R$ residual on data not used for identification.

the p.c.o MFD is not so easy to use for online parameter estimation. It was found that the two models represent nearly the same gain directionality.

\section{Discussion}

The validation results are compared for the black box and the process knowledge based approaches in table 3. The main difference between the two approaches is that the ARX structure obtained from the DYQUID is based upon process knowledge. This basis yields advantages in that it is possible to reason on the parameters in the model structure and to propose which additional parameters it may be worthwhile to consider if an improved model is desired. A second advantage of the DYQUID based ARX structure is that it can be expected to be reasonable within an operation region, where the assumptions behind the model structure development still are fulfilled. The disadvantages are that obtaining models from process knowledge are time consuming and that the parametrisation of monic ARX models are not understood yet, i.e. the model structure may not be identifiable. In contrast the advantage of the p.c.o. approach is that a model may be obtained with a very little effort without process knowledge, this model structure may also be reasonable at different operation regions, due to the fact that p.c.o. parametrieations are overlapping. The main disadvantage of p.c.o. models is that they do neither provide nor faciliate physical insight, and that they usually do not give monic input output models.

For linear noise free systems one might expect the condition number to grow to infinity when the true model order is exceeded, but as seen in table 2 no such change appers in the condition number calculated from the real data obtained from the distillation column. This is probably due to noise and nonlinearities.

A great deal of process knowledge should be used when measurements and actuators are selected. It seems not to be a prohibitively large effort to obtain models from process knowledge, compared to obtaining black box models, because the process understanding is already present.

\section{References}

[1] Andersen, H.W.; Kümmel, M.; Jørgensen, S.B. : Dynamics and Identification of a Binary Distillation Column. Chem. Eng. Sci. Vol 44, pp2571-2581 (1989).

[2] Bingulac, S.; Krtolica, R.K. : An algoritm for simultaneous order and parameter identification in multivariable systems, 8th IFAC/IF ORS Symposium on Identification and System Parameter Estimation Preprints, Beijing (1988).

[3] Corrêa, G.O.; Glover, K. : Pseudo-canonical Forms, Identifiable $P$ arametrisations and Simple Parameter Estimation for Linear Multivariable Systems: Input-Output Models, Automatica Vol 20, pp429-442 (1984).

[4] Gevers, M.; Wertz, V. : Techniques for selection of identifiable parametrizations for multivariable linear systems, Control and Dynamic Systems, Vol 24, Academic Press, New York, (1987).

[5] Guidorג, R.P.; Lonito, M.; Muratori, T. : The range error test in the structural identification of linear multivariable systems. IEEE Trans. Autom. Control AC-27,pp1044-54 (1982).

[6] Hallager, L.; Toftegárd, B., Clement, K., Jørgensen, S.B. : A distillation plant with an indirect heat pump for experimental studies of operation form, dynamics and control, IFAC Symposium on Dynamics and Control of Chemical Reactors and Distillation Columns, Bournemouth, England (1986).

[7] Jørgensen, S.B.; Goldsmidt, L.; Hallager, L. : Sparse process modelling for robust adaptive surveillance and control of a binary distillation column, IFAC Adaptive Control of Chemical Processes pp184 (1985).

[8] Jørgensen, S.B. : An approach to Multivariable Process Identification. IFAC Workshop on Model Based Control, Atlanta, Georgia (1988).

[9] Nielsen, C.S.; Andersen, H.W.; Brabrand, H.; Jørgensen, S.B. : Adaptive Dual Composition Control of a Binary Distillation Column with a Heat Pump. IFAC Adaptive Control of Chemical Processes, ADCHEM 88 (1988).

[10] Nielsen, C.S.; Andersen, H.W.; Brabrand, H.; Toftegärd, B.; Clement, K.; Jørgensen, S.B. : Identification and adaptive control of a binary distillation column with a heat pump. CHEMDATA 88, Göteburg June (1988).

[11] Pérez-Correa, J.R.; Kershenbaum, L.S. : State Space Formulation of Multivariable Self-Tuning Controllers, Chem. Eng. Res. Des. Vol 67, pp578588 (1989).

[12] Van Overbeek, A.J.M.; Ljung, L. : On-line parametrisation selection for multivariable system identification, Automatica Vol 18, pp529-543 (1982).

[13] Xiujing, W.; Zhongjun, Z.; Yushing, W. : A new on-line structure selection algoritm for MIMO state space models, 8th IFAC/IFORS Symposium on Identification and System Parameter Estimation Preprints, Beijing (1988). 